\title{
Surgical management and outcome of patients with thyroid disease during the COVID-19 pandemic
}

\author{
Y. Zhao (D) ${ }^{1}$, C. Jin ${ }^{1,2}$, Q. Song ${ }^{1,3}$, W. Kang ${ }^{1}$ and X. Xu (ID ${ }^{1, *}$ \\ ${ }^{1}$ Department of General Surgery, Peking Union Medical College Hospital, Chinese Academy of Medical Sciences and Peking Union Medical College, Beijing, China \\ ${ }^{2}$ Department of General Surgery, Huantai County People's Hospital, Qilu Hospital Huantai Branch, Shandong, China \\ ${ }^{3}$ Department of General Surgery, Beijing Longfu Hospital, Beijing, China
}

*Correspondence to: Department of General Surgery, Peking Union Medical College Hospital, Chinese Academy of Medical Sciences and Peking Union Medical College, Beijing, China (e-mail: xxq75@163.com)

\section{Dear Editor}

The COVID-19 pandemic has led to significant pressure on the healthcare system. An 80 per cent decrease in surgical activities and especially elective procedures has been suggested ${ }^{1,2}$. Some patients with thyroid nodules/cancers have experienced a more than 4-month postponement of their planned operation. Patients who are shown to have a risk of thyroid cancer based on a fineneedle aspiration (FNA) are thus faced with a risk of cancer progression and becoming increasingly dysphoric. A risk-group analysis was conducted at the authors' unit and, based on this, 50 thyroid operations undertaken during the pandemic. Before and during the hospitalization, measures were taken to protect both patients and healthcare workers ${ }^{3}$. Unexplained fever or any other symptoms linked to COVID-19 led to isolation in a separate room and immediate screening for SARS-CoV-2.

Patients were included prospectively from March to June 2020. They had to meet the following criteria: a FNA result indicating risk of cancer, aggressive signs, poorly controlled thyrotoxicosis, a benign nodule with compression and/or obvious increase in size, or patients with suspected carcinoma who were under enormous psychological stress. SPSS ${ }^{\circledR}$ version 19.0 (IBM, Armonk, New York, USA) was used for statistical analysis. Data are presented as median (i.q.r.) unless indicated otherwise.

Demographic and clinical characteristics, surgical management, and outcome of the 50 thyroid procedures are shown in Table 1. The interval from onset of diagnostic work-up was 8.0 (20.0) months and the waiting time before surgery (74 160) days. Twenty-five patients (50 per cent) had a FNA suspicious of cancer before surgery. Ten patients had suspected lymph node metastasis and nine had compressive symptoms. Most common comorbidities are shown in Table 1. In terms of surgical procedure, 18 patients had a hemithyroidectomy and 32 underwent total thyroidectomy, of which six procedures were combined with lateral lymph node dissection. Among those with a malignant diagnosis, 36 patients were diagnosed with papillary carcinoma and one had invasive squamous cell carcinoma. Among the 36 patients with papillary carcinoma, 17 had extrathyroidal extension and 15 had metastatic nodes. Thirteen patients had benign thyroid disorders on final pathology. There were no surgical complications or COVID-19 infections; the mean duration of hospital stay after surgery was 2.2 days.

Table 1 Demographic and clinical characteristics, surgical management, and outcome in 50 patients undergoing thyroid surgery

\begin{tabular}{|c|c|}
\hline & $\begin{array}{l}\text { No. of patients* } \\
\quad(n=50)\end{array}$ \\
\hline \multicolumn{2}{|l|}{ Demographic characteristics } \\
\hline Age $(\text { years) })^{\dagger}$ & $39: 11$ \\
\hline Sex ratio $(M: F)$ & $44.0(18.8)$ \\
\hline Duration of diagnostic work-up (months) ${ }^{\dagger}$ & $8.0(20.0)$ \\
\hline Waiting time before surgery (days) ${ }^{\dagger}$ & $74(160)$ \\
\hline \multicolumn{2}{|l|}{ Clinical characteristics } \\
\hline \multicolumn{2}{|l|}{ Triage to surgery } \\
\hline FNA & $25(50)$ \\
\hline Thyrotoxicosis & $0(0)$ \\
\hline Compressive symptoms & $9(18)$ \\
\hline Suspected lymph node metastasis & $10(20)$ \\
\hline \multicolumn{2}{|l|}{ Co-morbidities } \\
\hline Fatty liver or hyperlipidaemia & $6(12)$ \\
\hline Hypertension & $6(12)$ \\
\hline Hepatitis B & $6(12)$ \\
\hline Thyroid surgery history & $4(8)$ \\
\hline Diabetes mellitus & $2(4)$ \\
\hline Stroke & $2(4)$ \\
\hline \multicolumn{2}{|l|}{ Surgical procedure } \\
\hline Total thyroidectomy & $32(64)$ \\
\hline Hemithyroidectomy & $18(36)$ \\
\hline Lateral lymph node dissection & $6(12)$ \\
\hline \multicolumn{2}{|l|}{ Final pathology } \\
\hline Papillary carcinoma & $36(72)$ \\
\hline Benign thyroid disorders & $13(26)$ \\
\hline Invasive squamous cell carcinoma & $1(2)$ \\
\hline Extrathyroidal extension & $17(34)$ \\
\hline Lymph node metastasis & $15(30)$ \\
\hline \multicolumn{2}{|l|}{ Complications } \\
\hline Death & $0(0)$ \\
\hline Haemorrhage/haematoma & $0(0)$ \\
\hline Hoarseness & $0(0)$ \\
\hline Hypocalcaemia & $0(0)$ \\
\hline Wound infection & $0(0)$ \\
\hline COVID-19 infection & $0(0)$ \\
\hline
\end{tabular}

*With percentages in parentheses unless indicated otherwise;

${ }^{t}$ values are median (i.q.r.). FNA, fine-needle aspiration. 
Most thyroid cancers are indolent and so postponement of surgery in the era of COVID-19 has been suggested. There are limited data on the optimal postponement time. It has now been more than six months since the official announcement of the SARS-CoV-2 outbreak in China, and the WHO has expressed concerns that the virus may become endemic ${ }^{4}$. If deferral of elective surgery continues, especially in countries where COVID-19 is well controlled, not only will patients' distress worsen with time, but also surgeons will face stress and financial difficulties ${ }^{4}$. Moreover, a large and growing backlog of patients will be generated following COVID-19.

As the COVID-19 pandemic continues, more and more experience is being gained. Systematic and adequate infection control measures can be effective in COVID-19 prevention and in protecting patients 5 . Patients who undergo thyroid surgery have limited demand for ICU beds and blood transfusions. Most patients need only a short hospital stay and in this series no surgical complications were observed. Thus, with sufficient preoperative planning and preparation, elective thyroid surgery was safe and no patient or healthcare worker was confirmed to have COVID-19 infection. Care should be taken to balance the risk of COVID-19 infection against a sustainable resumption of elective procedures, and to take sufficient measures to keep patients and health workers safe from SARS-CoV-2.

\section{Acknowledgements}

Y.Z., C.J. and Q.S. contributed equally to this work.

Disclosure. The authors declare no conflict of interest.

\section{References}

1. Farid Y, Schettino M, Kapila AK, Hamdi M, Cuylits N, Wauthy P et al. Decrease in surgical activity in the COVID-19 pandemic: an economic crisis. Br J Surg 2020; https://doi.org/10.1002/bjs.11738

2. COVIDSurg Collaborative. Elective surgery cancellations due to the COVID-19 pandemic: global predictive modeling to inform surgical recovery plans. Br J Surg 2020;10.1002/bjs.11746

3. Zhao Y, Xu X. Thyroid surgery during COVID-19 pandemic: is it feasible? Br J Surg 2020; https://doi.org/10.1002/bjs.11867

4. Ghai $\mathrm{S}$. Will the guidelines and recommendations for surgery during COVID-19 pandemic still be valid if it becomes endemic? Int J Surg 2020;79:250-251

5. Meng L, Hua F, Bian Z. Coronavirus disease 2019 (COVID-19): emerging and future challenges for dental and oral medicine. $J$ Dent Res 2020;99: 481-487 